\title{
Characterization of the Regulatory Functions of Varicella-Zoster Virus Open Reading Frame 4 Gene Product
}

\author{
Defechereux P, Melen L, Baudoux L, Merville-Louis M-P, Rentier B,
} Piette J

Laboratory of Fundamental Virology, Department of Microbiology, Institute of Pathology B23, University of Liege, B-4000 Liege, Belgium

Varicella-zoster virus (VZV) open reading frame 4 (ORF4) encodes a protein with a predicted molecular weight of 51,540 presenting amino acid sequence homology with the immediate-early regulatory protein ICP27 of herpes simplex virus type 1. To investigate the regulatory properties of the ORF4 gene product, we performed a series of transient expression assays in Vero cells, using a plasmid expressing ORF4 as effector and several VZV genes and heterologous genes as targets. The VZV target plasmids contained promoter/regulatory regions from genes belonging to the three putative VZV kinetic classes fused to the chloramphenicol acetyltransferase (CAT) gene. The heterologous target plasmids consisted of promoter/regulatory regions of human cytomegalovirus, Rous sarcoma virus, and human immunodeficiency virus type 1 fused to the reporter gene. These experiments demonstrated that the ORF4 gene product activated expression of ORF62 in a dose-dependent fashion but had no effect on the expression of the three other putative immediate-early genes (ORF4, ORF61, and ORF63). When various amounts of ORF4 were transfected in the presence of early gene promoters, dosedependent transactivation was evidenced with the thymidine kinase gene (ORF36) and the major DNAbinding protein gene (ORF29) promoters; interestingly, little activity was detected with the promoter of the DNA polymerase gene (ORF28). No activation of late gene expression, represented by the glycoprotein I and glycoprotein II genes, was seen even over a wide range of concentrations of input ORF4 plasmid. Expression of pCMVCAT, pRSVCAT, and pHIVCAT was also stimulated by the ORF4 gene product. CAT mRNA analysis showed that activation of VZV target promoters occurs at the transcriptional and/or posttranscriptional level.

Varicella-zoster virus (VZV) is a neurotropic alphaherpes-virus which is responsible for two common, well-defined diseases: chickenpox, upon primary infection, and shingles, after reactivation of latent virus from the dorsal root ganglia. Classic studies of VZV biology have been severely hindered by the inability to produce high-titer cell-free virus with a good infectivity ratio (for reviews, see references 7 and 33). The determination of the entire nucleotide sequence of the VZV genome, however, has enabled structural and functional comparisons with herpes simplex virus type 1 (HSV-1), another intensively studied alphaherpesvirus $(8,9,12,13)$.

In the case of HSV-1, regulation of gene expression is ordered in a cascade fashion (17), implicating highly complex interactions between viral and cellular proteins. The immediate-early (IE, $\alpha$ ) genes are transcribed first following penetration of the virus in the absence of de novo protein synthesis $(2,4)$. A virion tegument protein, Vmw65 (VP16, $\alpha$-Tif), interacts with cellular factors, including the ubiquitous octamer-binding protein Oct-1, and forms protein-DNA complexes with the consensus sequence TAATGARAT located in the 5' promoter/regulatory regions of the five IE genes $(14,24,32,36)$. The net consequence is the stimulation of transcription by the host RNA polymerase II. Functional IE gene products are required for expression of later classes of genes (18). Early ( $\beta$ ) genes, encoding proteins necessary for DNA synthesis, are expressed next. Transcription of leaky-late $\left(\gamma_{1}\right)$ genes starts before viral DNA replication and peaks after DNA synthesis, whereas true-late $\left(\gamma_{2}\right)$ genes are transcribed after DNA synthesis only. Late genes encode virion structural proteins.

Insight into the functions of four of the five IE gene products of HSV-1 (i.e., ICPO, ICP4, ICP22, ICP27, and ICP47) has been gained by studies of numerous viral mutants and findings from transient assay experiments (for a review, see reference 11). Four VZV IE genes have been proposed as homologs of HSV-1 IE genes (i.e., open reading frame 4 [ORF4], ORF61, ORF62, and ORF63) (8, 33, 40). The ORF62 gene product, identified as a major regulatory protein $(3,9,10,19,35)$ that can complement the loss of ICP4 functions in HSV-1 mutants $(9,12)$, is present in the virion tegument $(22)$ and possesses DNA-binding properties somewhat similar to those of ICP4 (46). The ORF61 gene product has been confirmed as the functional homolog of ICPO (29) despite marked differences in amino acid sequences and in activities in transient assay experiments $(11,30,35)$. The ORF63 gene 
product (homologous to HSV-1 ICP22) shows frans-activating and trans-repressing effects on early and IE gene expression, respectively (21), indicating a potent role for this protein in the cascade of VZV gene expression. The ORF4 gene product $(51 \mathrm{kDa})$ presents a total amino acid sequence similarity of $28 \%$ with ICP27 $(63 \mathrm{kDa})$. The most similar region between the two proteins is the carboxyl-terminal portion (41\%), which encodes putative zinc finger metal-binding domains (45) and to which the ICP27 regulatory functions map $(16,28)$. ICP27 acts as a trans activator or as a trans repressor in combination with ICP4 and/or ICPO, depending on the target gene analyzed $(37,39,43)$. Recent work has shown that this protein acts partially posttranscriptionally to regulate HSV-1 gene expression, probably through mRNA processing $(27,38,41)$.

A

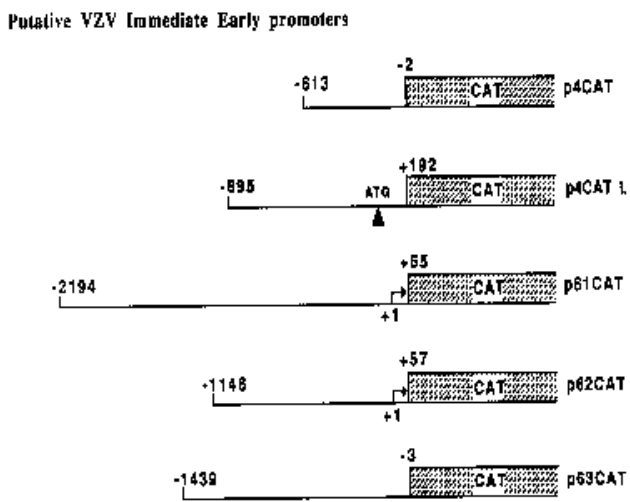

vit tarly promoters

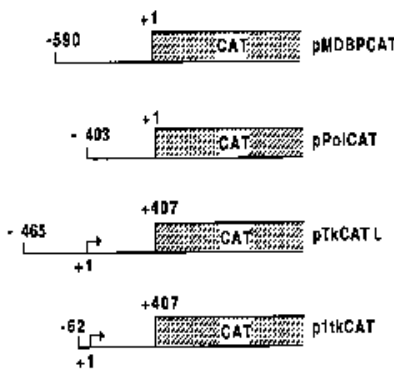

VZW 1,ate promoters

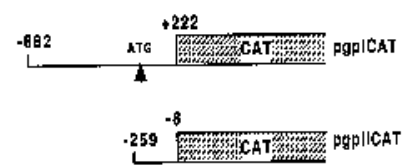

\section{B}

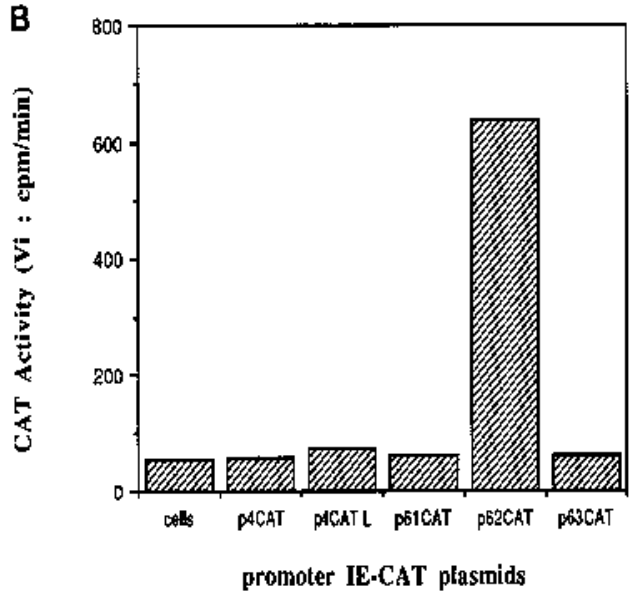

C

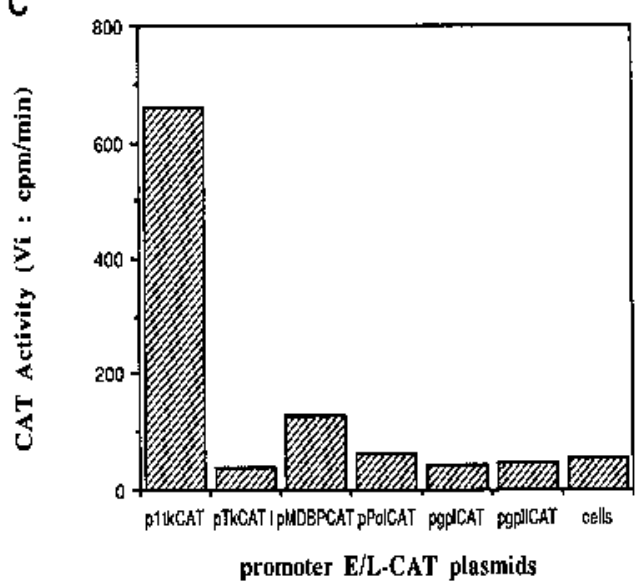

FIG. 1. (A) Schematic structures of the promoter/upstream regulatory element-CAT constructs used in this study. Restriction endonu-clease sites used in the construction of CAT constructs are indicated with their positions relative to the cap site (small arrows) or initiation ATG codon (large arrows). The large box represents the CAT coding sequence, and the solid lines represent VZV putative regulatory sequences. (B) Basal activities exhibited by VZV putative IE promoter/upstream regulatory elements fused to the CAT gene. Vero cells were transfected with $1 \mathrm{~g}$ of reporter CAT plasmid $\mathrm{p} 4 \mathrm{CAT}$, $\mathrm{p} 4 \mathrm{CAT}$, ( $p 4 C A T L), p \beta l C A T, p 62 C A T$, or p63CAT, using the lipofection technique. The total amount of DNA was equalized to $5 \mathrm{~g}$ with sonicated herring sperm DNA. Cells were harvested $44 \mathrm{~h}$ postlipofection, and CAT activity was assayed on $40 \mathrm{~g}$ of total cellular proteins. Initial rates (Vi) from chloramphenicol acetylation kinetics represent the promoters' intrinsic activities. Each transfection was repeated independently three times. (C) Basal activities exhibited by VZV putative early $(E)$ and late $(L)$ promoter/upstream regulatory elements fused to the CAT gene. Vero cells were cotransfected by lipofection with $0.5 \mathrm{~g}$ of pltkCAT or $1 \mathrm{~g}$ of pTkCAT ${ }_{\text {long }}$, pPolCAT, pMDBPCAT, pgpICAT, and pgpIICAT; CAT activities were assayed as described for panel $B$.

The ORF4 gene product enhances early gene expression and plays an accessory role in synergizing the effects of the ORF62 gene product $(19,35)$ on the expression of genes from the three kinetic classes. Unlike the case for its HSV-1 homolog, frans-repressing activities have not been associated with the 
ORF4 gene product $(35,37,39,43)$.

Thus, it appeared of interest to characterize further the regulatory functions of the ORF4 gene product and to define its role in the cascade of viral gene expression. Since VZV grows poorly in the available tissue culture systems, rendering the production of site-specific mutated viruses hazardous, we undertook transient assay experiments. Although this method cannot fully mimic the complex regulatory interactions that occur in VZV-infected cells, it provides an appropriate strategy for studying the regulatory properties of a given protein.

\section{Intrinsic activities of $\mathrm{VZV}$ promoters from the three putative kinetic classes in Vero cells.}

Analysis of the ORF4 gene product regulatory properties required VZV target genes whose expression could be monitored in the transfected cells; therefore, various promoter-chloramphenicol acetyltransferase (CAT) plasmids that carry promoter/regulatory regions from different VZV genes fused to the reporter CAT sequence were constructed (Fig. 1A). Construction of p62CAT, p4CAT ${ }_{\text {long, }}$, p1tkCAT, and pgpICAT has been described elsewhere $(19,26,30)$. Promoter sequences in p4CAT, p61CAT, p63CAT, pMDBPCAT, pPolCAT, and pgpIICAT result from classical cloning procedures (25). Vero cells at a density of $0.8 \times 10^{6}$ cells in 35 -mm-diameter six-well cluster dishes were transfected by the lipofection technique, using cationic vesicles of DOTAP (Boehringer, Mannheim, Germany). Various amounts of target constructs were prepared in 501 of Hanks buffered salt solution; the total amount of DNA in each experiment was kept constant by addition of sonicated herring sperm DNA. A solution of (N-[1-(2,3-dioleoyloxy)propyl]-N,N,N- trimethylammonium methylsulfate) was then added to a final concentration of $10 \mathrm{~g} / \mathrm{ml}$, and the mixtures were kept at room temperature for $10 \mathrm{~min}$ before being deposited on the cells.

CAT assay extracts were prepared 44 to $46 \mathrm{~h}$ after transfection, and CAT activities were determined essentially as described by Neumann et al. (31). The CAT activities were expressed as initial rates of chloramphenicol acetyla-tion reactions and were normalized with respect to the protein amount quantified by the Micro BCA protein assay reagent (Pierce, Rockford, I11.). The CAT activity measured by the diffusion assay was compared with that measured by the assay described by Gorman et al. (15), and the results were found to be equivalent (data not shown). Basal CAT activities associated with the 11 promoter-CAT constructs representing the different kinetic classes were assayed, and initial rates of chloramphenicol acetylation reactions are illustrated in Fig. 1B and C. Among the putative IE genes, ORF62 possess a high basal activity, whereas ORF4, ORF61, and ORF63 display very low levels of basal activity (Fig. IB). In the VZV early class, the thymidine kinase $(t k)$ gene (p1tkCAT) and the major DNA-binding protein (MDBP) gene (pMDBPCAT) show significant basal activity. Interestingly, a second construction containing a longer portion of the promoter/regulatory region of the $t k$ gene ( $\mathrm{pTkCAT}_{\text {long }}$ ) showed a low level of CAT activity. The promoter of the DNA polymerase gene (pPolCAT), another VZV putative early gene, also displayed low intrinsic activity (Fig. 1C). Both glycoprotein I and glycoprotein II genes (pgpICAT and pgpIICAT), constituting the putative late class representatives, are characterized by weak promoter activities (Fig. 1C). When titration experiments were performed, the only constructions which showed an increase in activity correlated with the increase in the transfected amounts were p62CAT, pMDBPCAT, and p1tkCAT (data not shown).

\section{Stimulation of VZV putative IE gene expression by the ORF4 gene product.}

Previous studies have proposed an accessory regulatory role for the ORF4 gene product $(19,35)$ in synergizing the effects of the ORF62 gene product. These studies focused only on a certain subset of promoters. We undertook an analysis of the effect of the ORF4 gene product alone on the expression of all four VZV putative IE genes in Vero cells. Cotransfection experiments were therefore carried out as described above, including this time in the transfection mixtures $1 \mathrm{~g}$ of each IE promoter target plasmid (i.e., p4CAT, p4CAT long, $_{\text {, }} 61 \mathrm{CAT}$, p62CAT, and p63CAT) (Fig. 1A) and increasing amounts of pGi4, a plasmid expressing ORF4 from its cognate promoter $(19,20)$.

To determine whether the concentration of effector plas-mid was critical to ORF4 action, we tested different input concentrations of pGi4. The smallest amount tested was $0.1 \mathrm{~g}$; since no activity was detected under such conditions (probably because of the weakness of the VZV promoter driving ORF4), we increased the input quantity to $0.5 \mathrm{~g}$ and then up to $7.5 \mathrm{~g}$. Figure $2 \mathrm{~A}$ illustrates the dosedependent activation of ORF62 gene promoter by pGi4, expressed as fold induction of CAT activity in the presence of the effector plasmid over uninduced target. pGi4 clearly elicited a dose-dependent 
activation of p62CAT, with a peak of CAT activity stimulation of 34-fold above the basal level of expression at 5.5 and $7.5 \mathrm{~g}$ of effector plasmid. However, pGi4 was unable to stimulate significantly any other putative IE promoter even in large molar excess (Fig. 2B). The absence of autoregulatory activity in our system and the dose-dependent activation indicate a possible weak interference from the ORF4 gene product onto its cognate promoter.
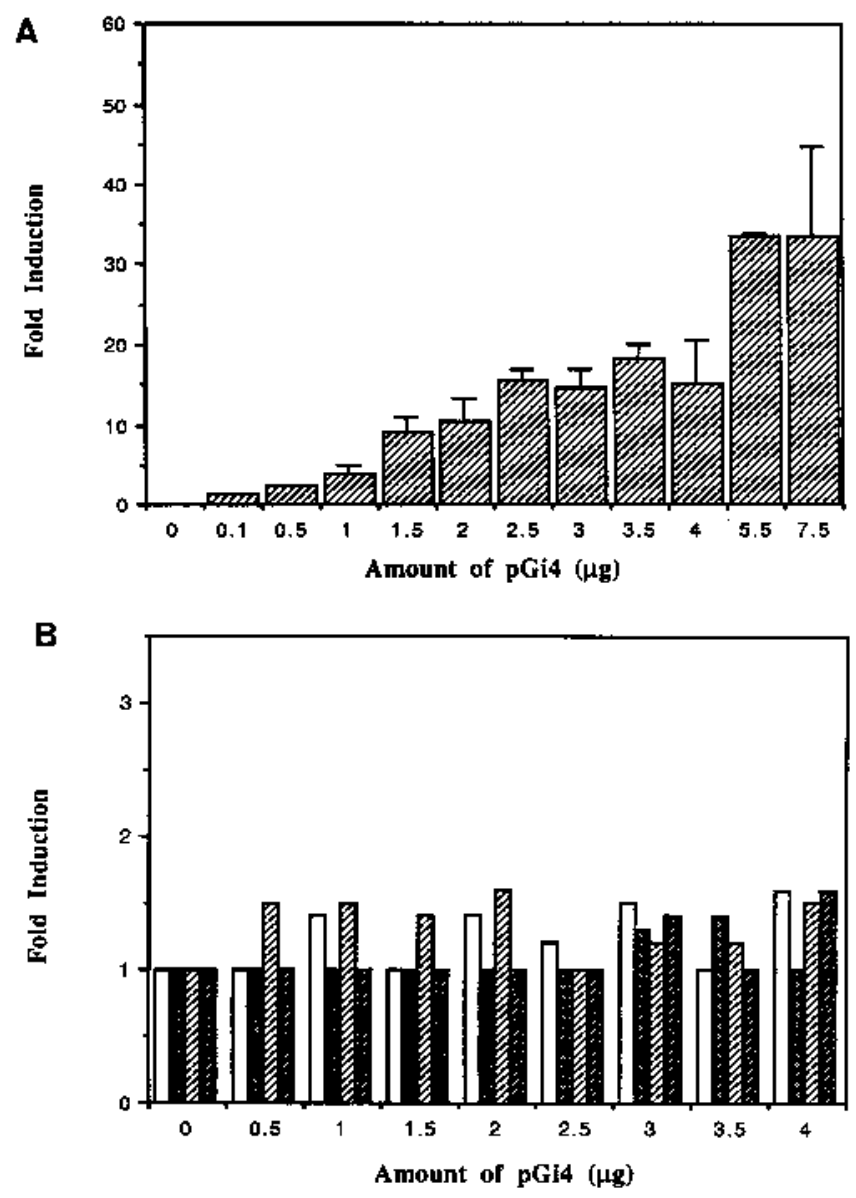

FIG. 2. Dose-dependent activation of the VZV putative IE promoters by the ORF4 gene product. (A) Vero cells were cotrans-fected with $1 \mathrm{~g}$ of p62CAT and increasing amounts of pGi4 (ORF4 plasmid) by lipofection. The total amount of DNA was maintained at 5 or $8.5 \mathrm{~g}$ with herring sperm DNA. $(B)$ Vero cells were cotrans-fected with 1 g of p4CAT( ), 1 g of p4CAT, long (H), 1 g of p61CAT(囫), or 1 g p63CAT ( ) and pGi4 in the indicated amounts. Total DNA quantity was equalized to $5 \mathrm{~g}$. Cells were harvested after $44 \mathrm{~h}$, and the CAT activities were assayed by using $40 \mathrm{~g}$ of total proteins for each condition. Initial rates of reaction quantitating CAT activity were calculated from kinetics, and data are presented as fold induction of CAT activity relative to the uninduced value, obtained with the reporter plasmid and herring sperm DNA alone in the absence of an effector plasmid, arbitrarily set to 1.0. Mean values and standard errors of the means from three different experiments are presented.

\section{Stimulation of VZV putative early and late genes by the ORF4 gene product.}

The ORF4 gene product is capable of stimulating the expression of the VZV tk gene (ORF36), a putative early gene, in Vero cells $(19,20,30)$ and, albeit to a lesser extent, in A3.01 cells, a human Tcell line (35). Cotransfection in Vero cells of increasing amounts of pGi4 over a 14-fold range with 0.5 $\mathrm{g}$ of $\mathrm{p} 1 \mathrm{tkCAT}$ confirmed the previous findings. Figure $3 \mathrm{~A}$ shows that such transactivation occurs in a dose-dependent fashion; maximal stimulations (55-fold) were obtained at higher concentrations in large molar excess of pGi4 (1:12.5 ratio), although clear transac-tivation could readily be achieved with $2 \mathrm{~g}$ of effector plasmid. 


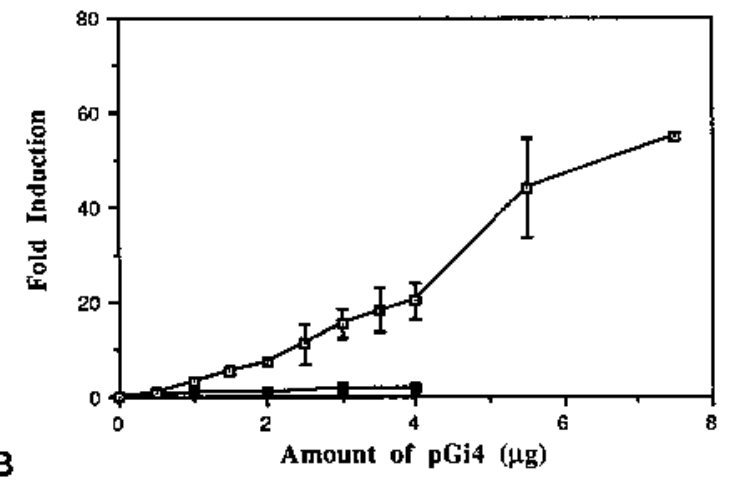

B
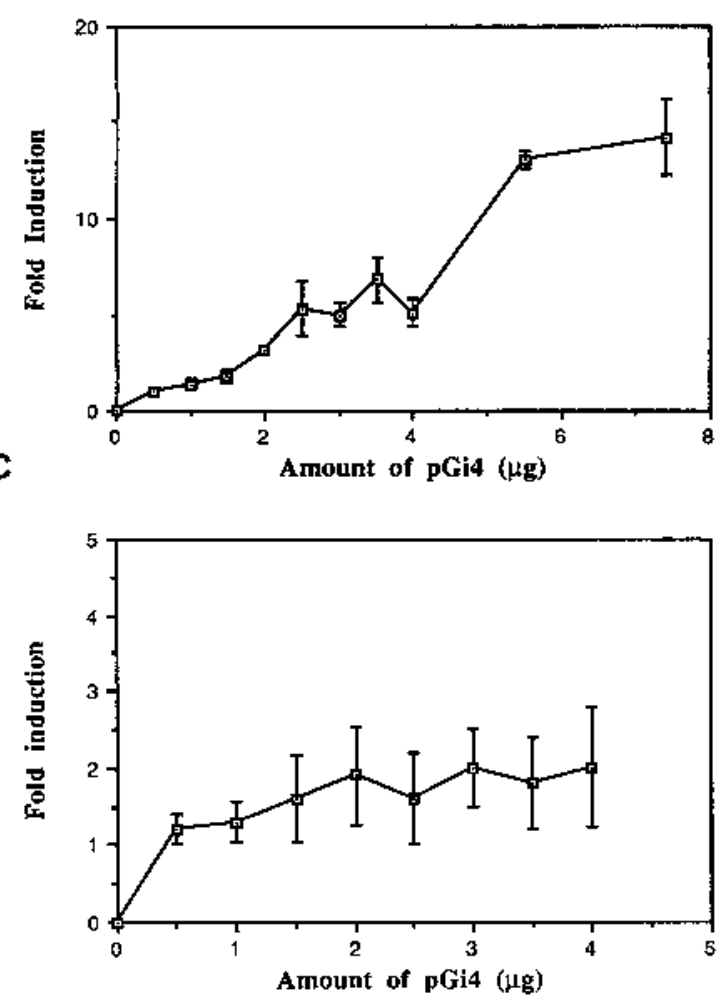

FIG. 3. Activation of putative VZV early promoters by the ORF4 gene product. (A) One-half microgram of pltkCAT ( ) or 1 g of pTkCAT, ${ }_{\text {long }}(\mathrm{O})$ was transfected in Vero cells along with the indicated amounts of pGi4; (B) 1 g of pMDBPCAT was cotrans-fected with increasing amounts of pGi4; (C) 1 of pPolCAT was cotransfected with $p$ Gi4 as indicated. The total amount of DNA was equalized to 5 and $8.5 \mathrm{~g}$ with herring sperm DNA. CAT activities were determined as described in the legend to Fig. 2; data are presented as fold induction of CAT activity relative to the activity associated with pltkCAT, pTkCAT, long, PMDBPCAT, or pPolCAT transfected in the absence of an effector plasmid. This uninduced value was arbitrarily set to 1.0. Four different experiments were conducted, and standard errors of the mean are shown as error bars.

Similar experiments conducted with $\mathrm{pTkCAT}_{\text {long, }}$, which carries a longer upstream regulatory region (465 to +407 relative to the mRNA cap site) than does p1tkCAT ( -62 to +407$)$ (Fig. LA), led to unexpected observations. p1tkCAT has a high basal activity in Vero cells; in contrast, the basal activity

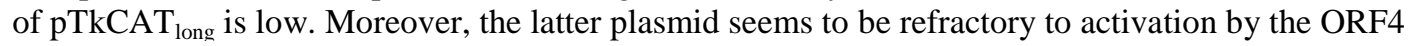
gene product, even at higher concentrations of input pGi4; only a twofold enhancement (probably not significant) could be achieved (Fig. 3A).

To investigate whether the ORF4 gene product could stimulate other putative early promoters, we constructed pMDBPCAT (with the promoter of ORF29 in front of the CAT cassette) and pPolCAT (with the promoter of ORF28 in front of the CAT cassette) (Fig. 1A). Cotransfection with increasing 
amounts of pGi4 demonstrated a clear dose-dependent transactivation of pMDBPCAT (up to 14-fold), whereas pPolCAT was induced only up to 2-fold in a non-dose-dependent fashion (Fig. 3B and C). Here, we provide evidence of stimulation of another putative early promoter by the ORF4 gene product.

Finally, the action of the ORF4 gene product on the expression of putative late genes was analyzed, as ORF4 seemed capable of stimulating activities of genes belonging to the two other kinetic classes. We used CAT constructs containing promoters of ORF68 (encoding glycoprotein I) and of ORF31 (encoding glycoprotein II). It was shown previously that ORF4 could transactivate pgpICAT in combination with ORF62 in Vero cells, and a twofold induction of CAT activity was detected with ORF4 alone in equimolar amounts (19); transfection of increasing amounts of pGi4 over a fourfold molar excess in the presence of $1 \mathrm{~g}$ of pgpICAT in Vero cells did not show any transactivation. Identical experiments conducted with pgpIICAT led to similar observations; no increase in CAT activity was associated with high concentrations of pGi4, although it would be interesting to increase the amounts of target DNA to determine whether the assay conditions are not limiting in the case of the late promoters.

\section{The ORF4 gene product acts at both transcriptional and posttranscriptional levels.}

Interestingly, the transient assay experiments indicated that the ORF4 gene product activated only promoters with relatively high basal activities. One explanation might be that the ORF4 gene product intervenes at a posttranscriptional step on preexisting mRNA, increasing its stability, 5' or 3' end processing, transport efficiency, or translation. To explore this possibility, slot blot analysis of steadystate levels of CAT mRNA was performed as described previously (21) to determine whether ORF4 action occurs at the transcriptional level.

Total cellular RNA was extracted by the guanidium isothiocyanate-cesium chloride method (6) from Vero cells transfected with either $0.5 \mathrm{~g}$ of p1tkCAT, $1 \mathrm{~g}$ of p62CAT, or $1 \mathrm{~g}$ of pMDBPCAT and pGi4 in increasing amounts to obtain significant stimulation. To quantify steady-state levels of CAT mRNA in the different transfection experiments, slot blots were analyzed with a PhosphorImager scanner and Image Quant software (Molecular Dynamics, Sunnyvale, Calif.). The fold stimulation in CAT activity was compared with the fold stimulation in CAT mRNA levels in each condition. Figure 4 shows that the dose-dependent stimulations cannot be strictly correlated with the increase in mRNA levels. CAT activity associated with p1tkCAT peaked in the presence of $5.5 \mathrm{~g}$ of pGi4 with a 60-fold stimulation; however, in the same condition, levels of CAT mRNA were only 7-fold higher. In the case of the ORF62 promoter, stimulation in CAT activity reached up to 45-fold with $7.5 \mathrm{~g}$ of pGi4; the mRNA levels in the same condition rose only 6-fold. Activity of pMDBPCAT was stimulated up to 16-fold in the presence of 7.5 g of effector plasmid; CAT mRNA levels were increased 10-fold. In the first two experiments, a striking discrepancy between the CAT activity and the CAT mRNA levels seems to exist. 
$\mathbf{A}$

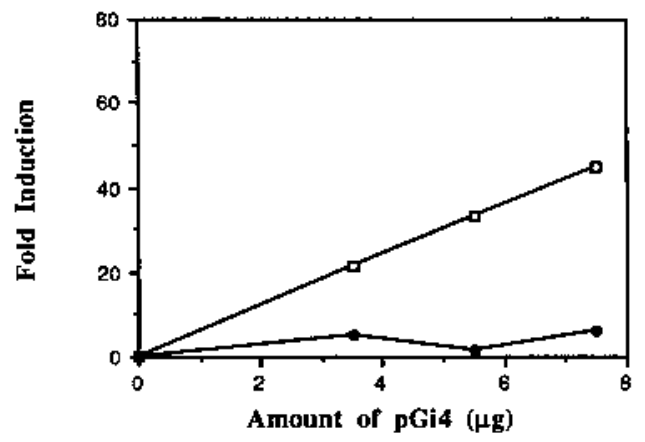

B

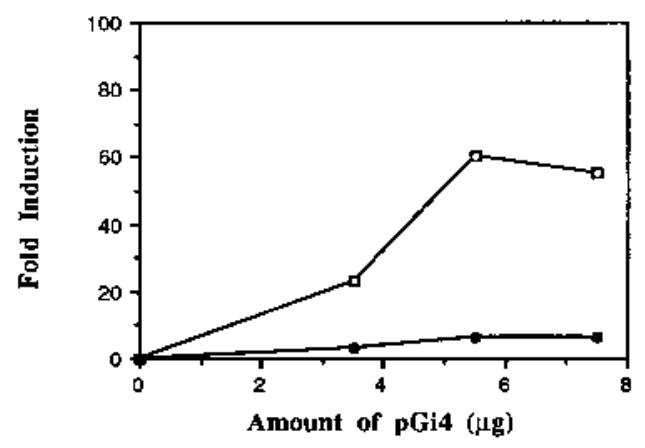

C

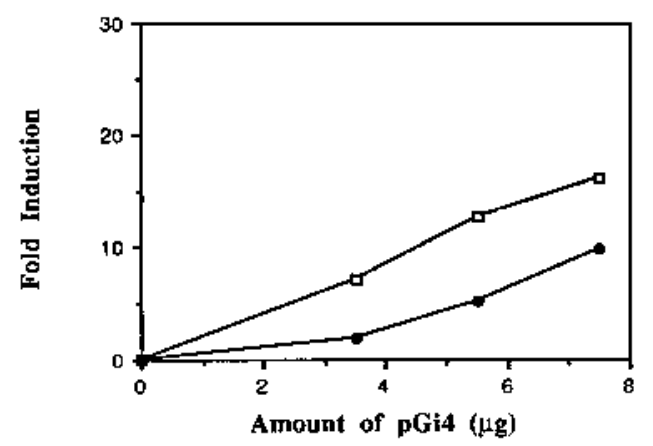

FIG. 4. Stimulation of CAT activity and CAT mRNA levels by the ORF4 gene product. Vero cells were cotransfected with $1 \mathrm{~g}$ of p62CAT (A), $0.5 \mathrm{~g}$ of pltkCAT $(B)$, or $1 \mathrm{~g}$ of pMDBPCAT $(C)$ and increasing amounts of $p G i 4$, expressing ORF4 under its cognate promoter. CAT activities were determined as described in the legend to Fig. 2; fold inductions ( ) are presented. Total RNA was extracted from transfected cells and slot blotted in four twofold dilutions. Hybridizations were carried out with a ${ }^{32} P$-labeled CAT probe, and RNA levels were quantified with a PhosphorImager scanner. Fold stimulation in CAT mRNA levels $(\bullet)$ is plotted.

In the case of the MDBP promoter, the difference turns out to be less pronounced.

Since we showed that the main property of the ORF4 gene product is to enhance activities of VZV promoters which possess basal activity in Vero cells (i.e., p62CAT, p1tkCAT, and pMDBPCAT), we investigated the possibility that the ORF4 gene product also activates heterologous viral promoters, which display high intrinsic activities in Vero cells. Therefore, Vero cells were cotransfected with either $0.5 \mathrm{~g}$ of pRSVCAT (CAT gene driven by the Rous sarcoma virus long terminal repeat) or $0.5 \mathrm{~g}$ of pCMVCAT (CAT gene driven by the promoter/enhancers of the IE region of cytomegalovirus) and 2 or 3 g of pGi4. pCMVCAT showed a higher basal activity than did pRSVCAT, but both constructs were stimulated up to sevenfold by ORF4. Cotrans-fection of $1 \mathrm{~g}$ of pHIVCAT (which carries the human immunodeficiency virus long terminal repeat) and 2 or $3 \mathrm{~g}$ of pGi4 elicited 3.5- and 8-fold stimulation of CAT activity, respectively (data not shown). These results 
taken together suggest that the ORF4 gene product can act in a transcriptional and/or posttranscriptional fashion.

To explore the molecular mechanisms which are responsible for the regulation of VZV gene expression, we examined the functions of the ORF4 gene product. We first analyzed the intrinsic activities of the target VZV promoters in Vero cells.

The ORF62 promoter was very effective at directing CAT production in Vero cells, in contrast to the results obtained in baby hamster kidney or HeLa cells (26). ORF36 displays a high basal activity in Vero cells, whereas its intrinsic activity is low in A3.01 cells (35). The difference in the detected activities might be due to the cell types used in the different studies. It is possible that a cell-specific factor(s) which interacts either directly with DNA sequences or with the general transcription machinery can account for the higher level of expression in Vero cells; another possibility is the presence of a repressor which shuts down the ORF62 and ORF36 promoter in certain cell types. We report for the first time that significant intrinsic activity is associated with the promoter of the MDBP gene (ORF29) in Vero cells but is not associated with the promoter of the DNA polymerase gene (ORF28); we also show that the ORF63 and ORF31 promoters have a very low level of basal activity. The other genes analyzed possess weak promoter activity in our system, consistent with previous reports $(19,23,35)$. Previous studies have shown that the ORF4 gene product enhances $t k$ gene expression and plays an accessory role in synergizing the effects of the ORF62 gene product (19, 35). We demonstrated that, unlike the findings of Perera et al. (35), the ORF4 gene product is capable, on its own in Vero cells, of activating in a dose-dependent fashion an IE promoter linked to the CAT gene, namely, the ORF62 promoter. The ORF63 promoter was not activated by the ORF4 gene product, even in the large concentration range that we tested; likewise, ORF4 and ORF61 were not activated as previously observed in A3.01 cells (35).

This lack of activity is not associated with the IE-CAT constructs, which carry regulatory sequences that extend largely over the identified initiation sites, respectively located between positions -34 and 54 (relative to the ATG codon) for ORF4, around positions -70 and -90 relative to the ORF63 ATG codon (21a), and at position -65 relative to the initiator codon of ORF61 (42). Moreover, these IE-CAT constructs are clearly stimulated by the ORF62 gene product (2a).

We showed that ORF4-mediated transactivation of the $t k$ gene takes place in a dose-dependent fashion in Vero cells. Despite the relatively short control sequence cloned in p1tkCAT (only 62 bp upstream of the mRNA cap site), this construct possesses a high basal activity in comparison with pTkCAT long, $_{\text {, }}$ which carries a longer upstream regulatory region (up to $-465 \mathrm{bp}$ ). The plasmid could hardly be stimulated by the 0RF4 gene product even in large molar excess; since the only difference between the two constructs is the length of the upstream region, it is possible that some negative cis elements are located in this sequence. Inchauspe et al. (19) had already observed such a difference, but the second $t k$-CAT plasmid used by these authors lacked a stretch of the long 5' nontranslated leader sequences (about 200 bp) that might be required for full transcriptional activity $(1,34)$. In our plasmids, the leader regions were identical; therefore, the loss of activity could not be attributed to a defect in mRNA stabilization or in specific sequences. Interestingly, expression from pTkCAT $\mathrm{p}_{\text {long }}$ could be readily stimulated by the ORF62 gene product (2a), demonstrating that pTkCAT $_{\text {long }}$ is a functional VZV promot-er-CAT construct.

Surprisingly, although the ORF29 promoter could clearly be stimulated by the ORF4 gene product, the other early target, ORF28, was not activated. The lengths of both promoter sequences cloned were chosen on a comparison basis with the $t k$ promoter; we expected them to include all essential elements, especially since the intergenic region between ORF28 and ORF29, which are transcribed in opposite directions, is very short, as is the case for the $t k$ gene (8).

The lower activities observed for the ORF28 promoter might be due to the shorter sequence cloned (400 bp) or to intrinsic structural differences. In keeping with published results obtained with other glycoprotein genes $(19,23,35)$, the ORF4 gene product did not activate expression from the glycoprotein II gene promoter. It stems from experimental evidence and from current knowledge about ORF4 that this polypeptide exerts its transactivating function on genes whose expression is significant in the transfected cells. This significant transcription is either achieved intrinsically or mediated by the strong transactivator ORF62 $(19,35)$.

Cotransfection experiments implicating ORF4- and ORF62-expressing plasmids and pMDBPCAT also indicated an enhancing action of the ORF4 gene product on the transactivation by ORF62 (data not shown). Thus, these results could indicate a possible posttranscriptional mechanism of regulation, the ORF4 gene product partly exerting its functions on the mRNA produced. The absence of direct correlation between detected CAT activities and mRNA steady-state levels in the cotransfected cells reinforces this idea. The observed increases in CAT mRNA levels could be due to an enhanced initiation, but this alone cannot account for the strong activation of CAT activities. More likely, the 
results reflect a better processing of the labile CAT mRNA (transport, stabilization, polyadenylation) or a more effective translation of equivalent level of messengers. A well-documented example of posttranscriptional control regulates the production of late adenovirus proteins; this mechanism involves preferential accumulation and translation of viral mRNA contributing to the shutoff of host protein synthesis during lytic infection (44). Such important posttranscrip-tional regulatory mechanisms have been recently attributed to the HSV-1 homolog $\operatorname{ICP} 27(38,41)$, which would explain its complex regulatory properties in HSV-1 gene expression. Moreover, ICP27 activates Moloney murine leukemia virus gene expression through a promoter-independent route (5).

Interestingly, the ORF4 gene product also showed a nonspecific ability to activate promoters from several other viruses, such as Rous sarcoma virus, human immunodeficiency virus type 1, and cytomegalovirus, which all display high activities in Vero cells. The constructs led to high levels of transcription, possibly allowing the ORF4 gene product to act on the preexisting mRNA. Transfection studies and analyses of viral mutants have shown that ICP27 is required for late gene expression; however, that has not been yet evidenced for the ORF4 gene product. Another difference between the VZV and HSV-1 proteins is the apparent lack of a negative regulatory function of the ORF4 gene product (35).

Elucidation of the mode of action of the ORF4 gene product will be made possible by confirming this hypothesis of posttranscriptional regulation, by exploring the functional similarities between the ORF4 gene product and ICP27 as well as identifying responsive elements. These experiments are currently in progress. Despite certain similarities between them, it is clear that VZV and HSV-1 employ distinct mechanisms to carry out their replicative cycles. Their regulatory proteins, among which is the ORF4 gene product, exhibit some properties in common but also some striking disparities that need to be further characterized. These observations might explain partially the unique biology of VZV and open many lines of investigation of this relatively recalcitrant virus.

We thank Giovanna Castellano for technical assistance and P. Kinchington for critical reading of the manuscript. Thanks to Charly Lambert and Pascal Lefebvre for the slot blot analysis. We are grateful to C. Preston, J. Ostrove, and P. Kinchington for providing the plasmids.

This work was supported by grants from the Belgian National Fund for Scientific Research (FNRS) (Brussels, Belgium), the Belgian National Lottery, and SmithKline Beecham Biologicals. P.D. is supported by a FNRS-SmithKline Beecham Biologicals grant. J.P. and M.-P.M.-L. are, respectively, senior research associate and research associate of the FNRS.

\section{References}

[1]. Ayer, D. E., and W. S. Dynan. 1988. Simian virus 40 late promoter: a novel tripartite structure that includes intragenic sequences. Mol. Cell. Biol. 8:2021-2033.

[2]. Batterson, W., and B. Roizman. 1983. Characterization of the herpes simplex virion-associated factor responsible for the induction of alpha genes. J. Virol. 46:371-377.

[2]a.Baudoux, L., et al. Personal communication.

[3]. Cabirac, G. F., R. Mahalingam, M. Wellish, and D. H. Gilden. 1990. Trans-activation of viral tk promoters by proteins encoded by varicella zoster virus open reading frames 61 and 62 . Virus Res. 15:57-68.

[4]. Campbell, M. E. M., J. W. Palfreyman, and C. M. Preston. 1984. Identification of herpes simplex virus DNA sequences which encode a trans-acting polypeptide responsible for stimulation of immediate-early transcription. J. Mol. Biol. 180:1-19.

[5]. Chapman, C. J., J. D. Harris, M.-A. Hardwicke, R. Sandri-Goldin, M. K. L. Collins, and D. S. Latchman. 1992. Promoter-independent activation of heterologous virus gene expression by the herpes simplex virus immediate-early protein ICP27. Virology 186:573-578.

[6]. Chirgwin, J. M., A. E. Przybyla, R. J. MacDonald, and W. J. Rutter. 1979. Isolation of biologically active ribonucleic acid from sources enriched in ribonuclease. Biochemistry 18:5294-5299.

[7]. Davison, A. J. 1991. Varicella-zoster virus. J. Gen. Virol. 72:475-486.

[8]. Davison, A. J., and J. E. Scott. 1986. The complete DNA sequence of varicella-zoster virus. J. Gen. Virol. 67:1759-1816.

[9]. Disney, G. H., and R. D. Everett. 1990. A herpes simplex virus type 1 recombinant with both copies of the Vmwl75 coding sequences replaced by the homologous varicella-zoster virus open reading frame. J. Gen. Virol. 71:2681-2689.

[10]. Disney, G. H., T. A. McKee, C. M. Preston, and R. D. Everett. 1990. The product of varicella- 
zoster virus gene 62 autoregu-lates its own promoter. J. Gen. Virol. 71:2999-3003.

[11]. Everett, R. D. 1987. The regulation of transcription of viral and cellular genes by herpesvirus immediate-early gene products.

Anticancer Res. 7:589-604.

[12]. Felser, J. M., P. R. Kinchington, G. Inchauspe, S. E. Straus, and J. M. Ostrove. 1988. Cell lines containing varicella-zoster virus open reading frame 62 and expressing the "IE" 175 protein complement ICP4 mutants of herpes simplex virus type 1. J. Virol. 62:2076-2082.

[13]. Felser, J. M., S. E. Straus, and J. M. Ostrove. 1987. Varicella-zoster virus complements herpes simplex virus type 1 temperature-sensitive mutants. J. Virol. 61:225-228.

[14]. Gerster, T., and R. G. Roeder. 1988. A herpes virus transacti-vating protein interacts with transcription factor OTF-1 and other cellular proteins. Proc. Natl. Acad. Sci. USA 85:6347-6351. [15]. Gorman, C. M., L. F. Moffat, and B. H. Howard. 1982. Recombinant genomes which express chloramphenicol acetyl-transferase in mammalian cells. Mol. Cell. Biol. 2:1044-1051.

[16]. Hardwicke, M. A., P. J. Vaughan, R. E. Sekulovich, R. O'Con-ner, and R. M. Sandri-Goldin. 1989. The regions important for the activator and repressor functions of herpes simplex virus type $1 \alpha$ protein ICP27 map to the C-terminal half of the molecule. J. Virol. 63:4590-4602.

[17]. Honess, R. W., and B. Roizman. 1974. Regulation of herpesvirus macromolecular synthesis. I. Cascade regulation of the synthesis of three groups of viral proteins. J. Virol. 14:8-19.

[18]. Honess, R. W., and B. Roizman. 1975. Regulation of herpesvirus macromolecular synthesis: sequential transition of polypeptide synthesis requires functional polypeptides. Proc. Natl. Acad. Sci. USA 72:1276-1280.

[19]. Inchauspe, G., S. Nagpal, and J. M. Ostrove. 1989. Mapping of two varicella-zoster virusencoded genes that activate the expression of viral early and late genes. Virology 173:700-709. [20]. Inchauspe, G., and J. M. Ostrove. 1989. Differential regulation by varicella-zoster virus (VZV) and herpes simplex virus type-1 trans-activating genes. Virology 173:710-714.

[21]. Jackers, P., P. Defechereux, L. Baudoux, C. Lambert, M. Massaer, M. P. Merville-Louis, B. Rentier, and J. Piette. 1992. Characterization of regulatory functions of the varicella-zoster virus gene 63-encoded protein. J. Virol. 66:3899-3903.

[21a].Kinchington, P. Personal communication.

[22]. Kinchington, P. R., J. K. Houghland, A. M. Arvin, W. T. Ruyechan, and J. Hay. 1992. The varicella-zoster virus immediate-early protein IE62 is a major component of virus particles. J. Virol. 66:359-366.

[23]. Ling, P., P. R. Kinchington, M. Sadeghi-Zadeh, W. T. Ruyechan, and J. Hay. 1992. Transcription from varicella-zoster virus gene 67 (glycoprotein IV). J. Virol. 66:3690-3698.

[24]. Mackem, S., and B. Roizman. 1982. Structural features of the herpes simplex virus $\alpha$ gene 4,0 , and 27 promoter-regulatory sequences which confer $\alpha$ regulation on chimeric thymidine kinase genes. J. Virol. 44:939-949.

[25]. Maniatis, T., £. F. Fritsch, and J. Sambrook. 1982. Molecular cloning: a laboratory manual. Cold Spring Harbor Laboratory, Cold Spring Harbor, N.Y.

[26]. McKee, T. A., G. H. Disney, R. D. Everett, and C. M. Preston. 1990. Control of expression of the varicella-zoster virus major immediate early gene. J. Gen. Virol. 71:897-906.

[27]. McLaughlan, J., A. Phelan, C. Loney, R. M. Sandri-Goldin, and J. B. Clements. 1992. Herpes simplex virus IE63 acts at the posttranscriptional level to stimulate viral mRNA 3' processing. J. Virol. 66:6939-6945.

[28]. McMahan, L., and P. A. Shaffer. 1990. The repressing and enhancing functions of the herpes simplex virus regulatory protein ICP27 map to C-terminal regions and are required to modulate viral gene expression very early in infection. J. Virol. 64:3471-3485.

[29]. Moriuchi, H., M. Moriuchi, H. A. Smith, S. E. Strauss, and J. I. Cohen. 1992. Varicella-zoster virus open reading frame 61 protein is functionally homologous to herpes simplex virus type 1 ICP0. J. Virol. 66:7303-7308.

[30]. Nagpal, S., and J. M. Ostrove. 1991. Characterization of a potent varicella-zoster virus encoded trans-repressor. J. Virol. 65:5289-5296.

[31]. Neumann, J. R., C. A. Morency, and K. O. Russian. 1987. A novel rapid assay for chloramphenicol acetyltransferase gene expression. BioTechniques 5:444.

[32]. O'Hare, P., and C. R. Goding. 1988. Herpes simplex virus regulatory elements and the immunoglobulin octamer domain bind a common factor and are both targets for virion transacti-vation. Cell 52:453-445.

[33]. Ostrove, J. M. 1990. Molecular biology of varicella-zoster virus. Adv. Virus Res. 38:45-99.

[34]. Pederson, N. E., S. Person, and F. Homa. 1992. Analysis of the gB promoter of herpes simplex virus type 1: high-level expression requires both an 89-base-pair promoter fragment and a 
nontranslated leader sequence. J. Virol. 66:6226-6232.

[35]. Perera, L. P., J. D. Mosca, W. T. Ruyechan, and J. Hay. 1992. Regulation of varicella-zoster virus gene expression in human T lymphocytes. J. Virol. 66:5298-5304.

[36]. Preston, C. M., M. C. Frame, and M. E. M. Campbell. 1988. A complex formed between cell components and an HSV structural polypeptide binds to a viral immediate early gene regulatory DNA sequence. Cell 52:425-434.

[37]. Rice, S. A., and D. M. Knipe. 1990. Genetic evidence for two distinct transactivation function of the herpes simplex virus $\alpha$ protein ICP27. J. Virol. 64:1704-1715.

[38]. Sandri-Goldin, R. M., and G. E. Mendoza. 1992. A herpesvirus regulatory protein appears to act post-transcriptionally by affecting mRNA processing. Genes Dev. 6:848-863.

[39]. Sekulovich, R. E., K. Leary, and R. M. Sandri-Goldin. 1988. The herpes simplex virus type $1 \alpha$ protein ICP27 can act as a trans-repressor or a trans-activator in combination with ICP4 and ICP0. J. Virol. 62:4510-4522.

[40]. Shiraki, K., and R. W. Hyman. 1987. The immediate early proteins of varicella zoster virus. Virology 156:423-426.

[41]. Smith, I. L., M. A. Hardwicke, and R. M. Sandri-Goldin. 1992. Evidence that the herpes simplex virus immediate early protein ICP27 acts post-transcriptionally during infection to regulate gene expression. Virology 186:74-86.

[42]. Stevenson, D., K. L. Colman, and A. J. Davison. 1992. Characterization of the varicella-zoster virus gene 61 protein. J. Gen. Virol. 73:521-530.

[43]. Su, L., and D. M. Knipe. 1989. Herpes simplex virus $a$ protein ICP27 can inhibit or augment viral gene transactivation. Virology 170:496-504.

[44]. Tevethia, M. J., and D. J. Spector. 1989. Heterologous transac-tivation among viruses. Prog. Med. Virol. 36:120-190.

[45]. Vaughan, P. J., K. J. Thibault, M. A. Hardwicke, and R. M. Sandri-Goldin. 1992. The herpes simplex virus immediate early protein ICP27 encodes a potential metal-binding domain and binds Zinc in vitro. Virology 189:377-384.

[46]. Wu, C. L., and K. W. Wilcox. 1991. The conserved DNA-binding domains encoded by the herpes simplex virus type 1 ICP4, pseudorabies virus IE180, and varicella-zoster virus ORF62 genes recognize similar sites in the corresponding promoters. J. Virol. 65:1149-1159. 